\title{
Scholarly

\section{Extranodal NK/T-Cell Lymphoma without Nasal Cavity Involvement Associated with Epstein-Barr Virus: A Multimodality-Based Diagnosis of Two Cases}

\author{
Fernando Cobo, ${ }^{1}$ Clara Chamorro, ${ }^{1}$ Francisco Ramos, ${ }^{2}$ and Ángel Concha ${ }^{1}$ \\ ${ }^{1}$ Infectious Pathology Unit, Department of Pathology and Tissue and Tumor Bank, Hospital Universitario Virgen de las Nieves, \\ 18014 Granada, Spain \\ ${ }^{2}$ Department of Pathology, Hospital de Torrecárdenas, 04009 Almería, Spain
}

Correspondence should be addressed to Fernando Cobo, fernando.cobo.sspa@juntadeandalucia.es

Received 5 January 2009; Accepted 5 February 2009

\begin{abstract}
We report two cases of extranodal NK/T-cell lymphoma, nasal type, in immunocompetent patients without nasal cavity involvement. In the two cases, the initial presumptive diagnosis was tuberculosis and there was a rapid dissemination of the tumor with short survival after the hospital admittance. An autopsy was performed showing infiltration in several organs including lymph nodes and mesenteric and retroperitoneal fat. Histological sections showed an angiocentric and angiodestructive growth pattern and the immunophenotype was CD45+, CD3+ (cytoplasmic), as well as Granzyme B+ and EBV+. However, CD56 expression was only positive in a case in which the molecular study showed T-cell gene rearrangement with monoclonal appearance and associated with hemophagocytic syndrome. These cases represent rare examples of NK/T-cell lymphoma disseminated outside the nasal cavity highly aggressive that lead to the rapid death of the patients.
\end{abstract}

Copyright (C) 2009 Fernando Cobo et al. This is an open access article distributed under the Creative Commons Attribution License, which permits unrestricted use, distribution, and reproduction in any medium, provided the original work is properly cited.

\section{Introduction}

Extranodal NK/T-cell lymphomas are a group of neoplasias characterized by a broad morphologic spectrum. These tumors mainly show an extranodal presentation such as nasal cavity and nasopharynx, but may have other sites of involvement. Extranodal NK/T-cell lymphomas occur most commonly in South East Asia and South America and are rare elsewhere [1].

These lymphomas are more frequent in immunocompetent patients though have also been described in immunosuppressed patients $[2,3]$. The etiology of this kind of lymphoma is unknown but NK/T-cell lymphomas of nasal type are constantly associated with EBV irrespective of the geographic origin [1]; although EBV has a special tropism with B-cell type, this virus can infect $\mathrm{T}$ and $\mathrm{NK}$ cells and this infection leads to a higher risk of lymphoma [4]. Although in Asian populations the association of NK/T-cell lymphomas extending outside the nasal cavity with EBV is strong, this association seems less clear in white populations
[1]. EBV probably has an oncogenic role in these lymphomas but the mechanisms by which EBV can produce the cell transformation or gain entry into tumor cells of NK/T-cell lymphomas are unknown.

The histological features of these tumors are often a diffuse angiocentric infiltrate, with necrosis and vascular destruction [1]; however, these histological characteristics might be seen in other types of lymphoma [5]. Characteristically, NK/T-cell lymphomas express CD2, CD56, and intracellular $\mathrm{CD} 3 \varepsilon$, and might produce cytotoxins perforin, granzyme B, or TIA-1 [6], but there are cases with CD56cytotoxic T-cell phenotype [1]. The clinical features are those related with the affected organ, but the disease might quickly disseminate to several locations such as gastrointestinal tract and skin, and in some cases, may be complicated by a hemophagocytic syndrome $[7,8]$.

The detection of monoclonal expansion of the TCR $\gamma$ chain genes can be an important supplement for the diagnosis of NK/T-cell lymphomas. In the nasal type, NK/Tcell lymphoma clonal T-cell receptor gene rearrangement 
has been reported in several cases with molecular methods obtaining different detection rates [9].

In this manuscript, we have described two recent cases of extranodal NK/T-cell lymphoma in white patients without nasal cavity involvement, $\mathrm{EBV}+$, dissemination to several organs with important infiltration of mesentericintraperitoneal fat tissue, quick fatal progression, and presumptive clinical diagnosis of tuberculosis. The final diagnosis of these cases was performed by means of multiple approaches correlating clinical and morphological, immunophenotypic, molecular data from the autopsy.

\section{Case Reports}

Case 1. A 61-year-old Spanish man was admitted to hospital due to the presence of fever with pancytopenia 5 months before this visit. The patient had been diagnosed with diabetes mellitus type 2 eight years ago. At presentation, his temperature was $39.5^{\circ} \mathrm{C}$, blood pressure was $90 / 55 \mathrm{mmHg}$ and pulse was 108/min. Physical examination showed hepatosplenomegaly and the rest of the physical examination was unremarkable. The initial presumptive diagnosis was an infection (e.g., disseminated tuberculosis) or neoplasia because the Ca 19.9 level was elevated. A thoracoabdominal CT scan was performed and showed hepatomegaly, ascitic fluid, and bilateral pleural effusion. A bone marrow biopsy was also carried out showing presence of haemophagocytic histiocytes infiltration. Serologies for HBV, HCV, HIV, CMV, EBV, Leishmania, and Toxoplasmosis were carried out but were normal.

On day 15 of the admittance, the patient developed progressive dyspnea and multiorgan failure leading to death. An autopsy was later performed. The main macroscopic findings were several necrotic-hemorrhagic nodules in the mesenteric-intraperitoneal fat (Figure 1(a)) and multiple necrotic foci in lung, liver, kidney, and suprarenal (Figure 1(b)). Congestive lung parenchyma with the presence of pleural effusion as well as the presence of pericardial effusion also was observed. Hepatomegaly with ascitic fluid in peritoneal cavity and congestive splenic parenchyma was discovered. The rest of the organs were normal, including the brain and the nasal cavity.

Multiple samples of tissues from all these organs were taken and histological sections were stained with hematoxylin eosin and also immunostains were carried out for different primary antibodies for immunophenotyping the cellular infiltrate. The microscopic study showed liver, spleen, lung, pancreas, bone marrow, and suprarenal infiltration by a lymphoproliferative infiltrate, as well as signs of haemophagocytic syndrome. The infiltrate was predominantly angiocentric, with necrosis and vascular destruction (Figures 1(c) and 1(d)).

The immunochemical study was performed using reagents and materials provided by Dako Diagnostic (Carpinteria, Calif, USA) and Novocastra (Newcastle, UK) and used following the manufacturer's recommended specifications and by means of the automatic immunostainer Horizon (Dako Diagnostic, Glostrup, Denmark).
The immunophenotype of this tumor was CD45+, $\mathrm{CD} 2+, \mathrm{CD} 3 \varepsilon+, \mathrm{CD} 56+$ (Figure 1(e)), Granzyme B+ and TIA+ (Figure 1(f)), as well as CD20-, CD79a-, CD43- and CD45Ro-. The abundant histiocytic infiltrate was CD68+.

A PCR study for EBV viral genome of Bam-HI-W system was performed and the result was positive. This was also corroborated by the LMP1 immunochemistry for EBV.

Finally, a multiplex real-time PCR study for T-cell gene rearrangement was performed on DNA extracted from the paraffin block. A capillary electrophoresis in polyacrylamide gel by means of the ABI-PRISM 310 genetic analyzer (Applied Biosystems, foster City, Calif, USA) was carried out. This study showed T-cell gene rearrangement with monoclonal appearance.

Case 2. A 46-year-old Spanish man was diagnosed with ulcerative colitis in 2003, and with treatment with azathioprine $(200 \mathrm{mg} /$ day) since February 2005. In December 2006, the patient was admitted to hospital due to the presence of arthralgias and fever. The physical examination was unremarkable and treatment with prednisone was started. A few days later, the patient had general disturbance with neurological symptomatology (facial paralysis, loss of strength) and loss of consciousness. A brain CT scan was performed showing images that could seem a meningoencephalitis of probable tuberculous or cryptoccocal etiology. The cerebrospinal fluid analysis showed an elevation of the pressure and the leucocytes ( $80 \%$ lymphocytes). The rest of the studies were unremarkable. A treatment with antituberculous, antifungal, and antibiotic drugs began, but the symptoms did not remit, and in February 2007, the patient died. An autopsy was also performed. As in Case 1, the main macroscopic findings were necrotichemorrhagic nodules of the mesenteric-intraperitoneal fat tissue (Figure 2(a)). Other findings were as follows: plentiful pleural effusion with turbidity, several pleuro-pleural adherences, and acute haemorrhagic congestive of both lungs; plentiful ascitic fluid with turbidity, with several peritonealmesenteric adherences and multiple enlarged mesenteric, peripancreatic and paraaortic lymph nodes; hepatomegaly with necrotic nodular parenchymal lesion of $1 \mathrm{~cm}$; colonic mucosa with multiple ulcerative lesions and multiple mesenteric adenopathies; heart appearing with thickness of perimiocardium and important infiltration of the fat (Figure 2(b)); both kidneys had multiple cortical necrotic lesions; as well as a cerebrospinal fluid with turbidity and thick meninges were observed. There was no malignant infiltration in the nasal cavity. A histological examination was performed and microscopic slides of all organs were stained with hematoxylin eosin and also immunostains were carried out for different primary antibodies to immunophenotype the cellular infiltrate. The meninges' alteration was induced by unspecific inflammation but there was no evidence of neoplastic infiltration. The microscopic examination showed renal, liver, and intestinal infiltrations as well as evidence of mesenteric-intraperitoneal fat infiltration by neoplastic cells (Figures 2(c) and 2(d)).

The immunochemical study was performed using reagents and materials provided by Dako Diagnostic 


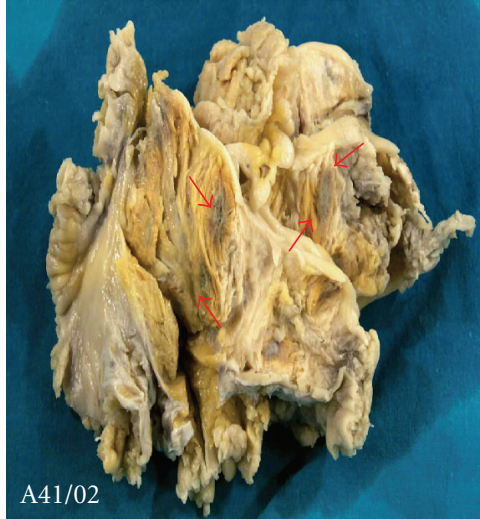

(a) An image showing abundant necrotichemorrhagic nodules corresponding to mesenteric-retroperitoneal fat tissue (see the red arrows)

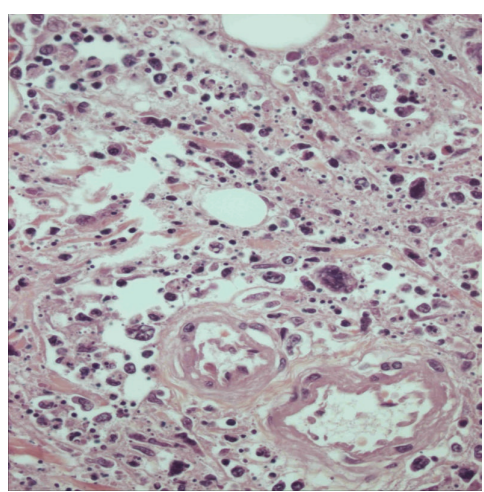

(d) Fibrinoid changes in vascular walls and abundant necrotic cellular debris. Note the isolated pleomorphic nuclei of neoplastic cells

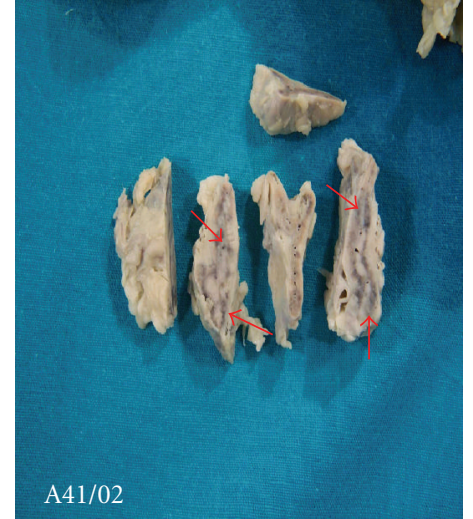

(b) A photograph showing the important suprarenal infiltration for the lymphomatous cells (see the red arrows)

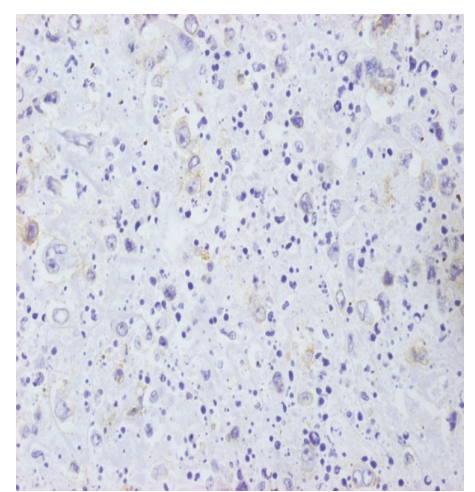

(e) Expression of the CD56 marker in

the malignant cells

Figure 1: (Case 1).

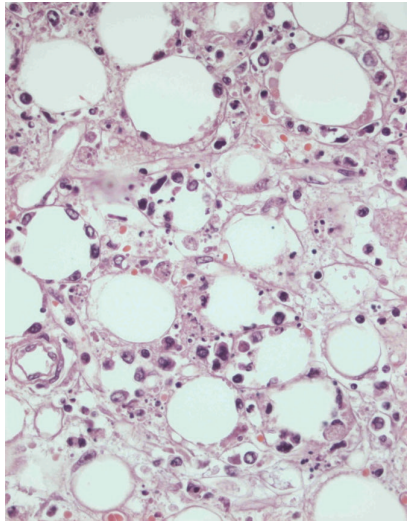

(c) Polymorphous lymphocytic infiltrate of fat tissue with atypical elements

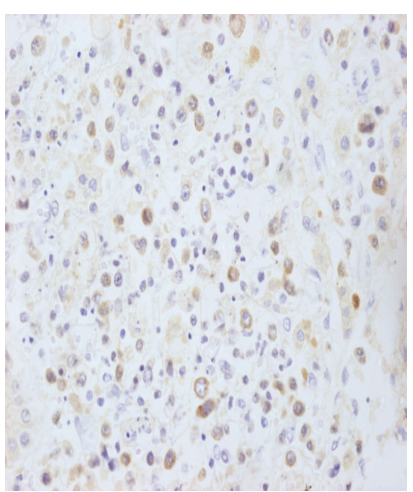

(f) The neoplastic cells also show TIA expression in the malignant cells
(Carpinteria, Calif, USA) and Novocastra (Newcastle, UK) and used following the manufacturer's recommended specifications and by means of the automatic immunostainer Horizon (Dako Diagnostic).

The immunophenotype of this tumor was CD45+, $\mathrm{CD} 2+, \mathrm{CD} 3 \varepsilon+$, granzyme B (Figure 2(e)), and CD56-, CD20-, CD4-, CD8-, CD72a-. EBER-EBV in situ hybridization was also positive (Figure $2(\mathrm{f})$ ) as well as LMP-1 immunochemistry for EBV (figure not shown).

\section{Discussion}

Extranodal NK/T-cell lymphoma, nasal type, is a lymphoproliferative disorder characterized, in the majority of cases, by an angiocentric infiltrate with vascular destruction and necrosis [1]. In these kinds of lymphomas, the etiology is still unkown, but they are always associated with EBV, irrespective of the geographic origin [1]. These tumors are more frequent in Asian and South American populations and are rare in Western countries; however, in our hospital, we have recently diagnosed three cases, two of them are described in this report and the other has already been published [3].

The most common location of NK/T-cell lymphoma is the nasal cavity although some cases might be located in other places and lymph nodes also might be infiltrated. Curiously, in our cases, the most important affectation was observed in lymph nodes and in retroperitoneal and mesentery fat tissue as well as abdominal organs. In both cases, massive infiltration of retroperitoneal fat tissue and mesentery with pancreatic involvement was demonstrated.

Another important unexpected fact is that in our two cases there was no nasal cavity affectation like in the case we recently published [3]. For this reason, we can speculate the possibility of less nasal cavity affectation and more abdominal involvement in extranodal NK/T-cell lymphomas originated from patients in western countries.

On the other hand, a few reports have shown the association of extranodal NK/T-cell lymphoma with haemophagocytic syndrome $[7,8]$. This complication is characterized by the presence of fever, pancytopenia, and hemophagocytic 


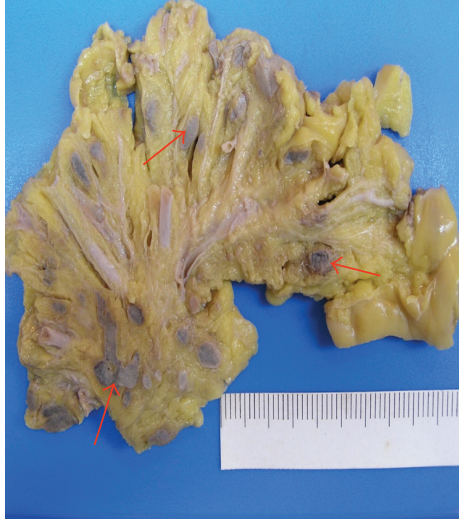

(a) Similar image to Figure 1(b) showing abundant necrotic-hemorrhagic nodules corresponding to the mesenteric fat tissue with enlargement of lymph nodes (see the red arrows)

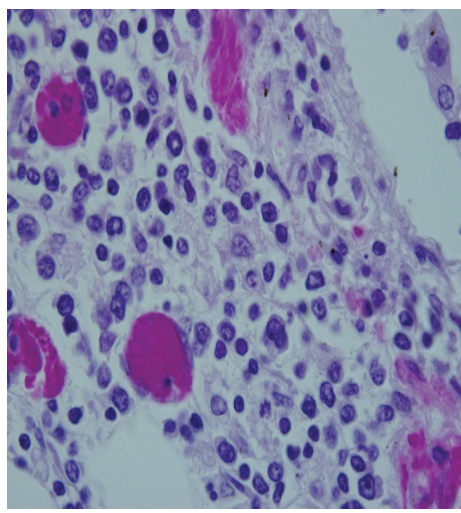

(d) High magnification of polymorphic cellular population with occasional pleomorphic nuclei

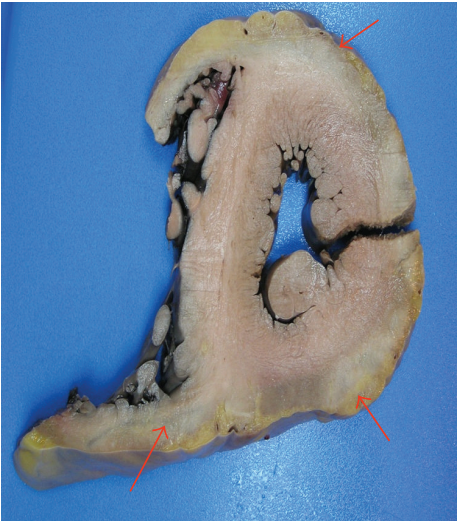

(b) Macroscopic heart infiltration visualized as white indurate nodules more evident in the pericardium fat and in the superficial ventricular myocardium (see the red arrows)

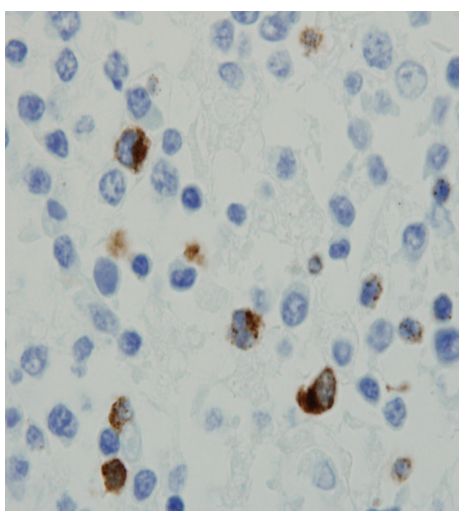

(e) There is a cytoplasmic-granular positivity in the malignant cells for granzyme $\mathrm{B}$ (cytotoxic molecules)

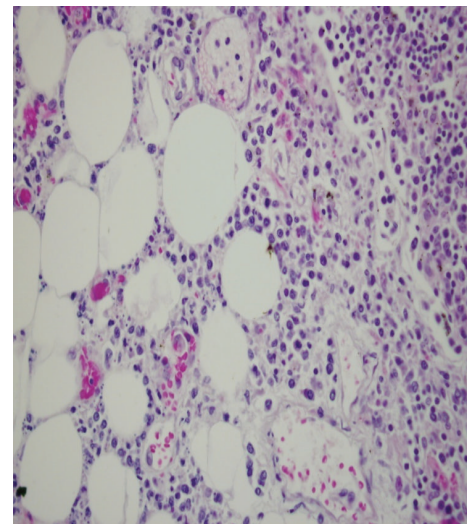

(c) Diffused infiltration of fat tissue by atypical lymphocytes

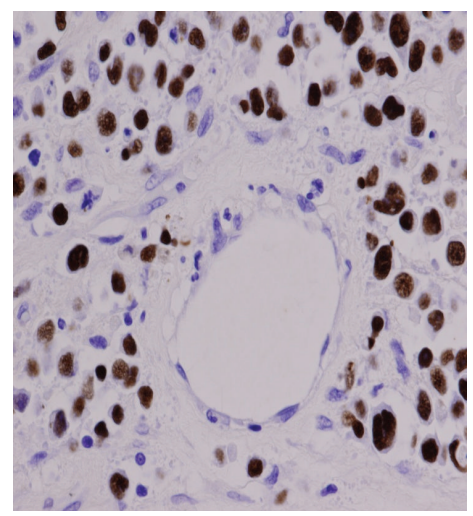

(f) The EBER-EBV in situ hybridization appears positive in numerous tumoral cells

Figure 2: (Case 2).

histiocytes in bone marrow. In Case 1, a hemophagocytic syndrome and bone marrow affectation could be seen.

At present, the diagnosis of these kinds of lymphomas is made with greater precision by using immunophenotyping [6]. Extranodal NK/T-cell lymphoma characteristically express T-cell marker CD2, NK cell marker CD56, and lack surface CD3 expression (but express intracellular CD3E). Also, this tumor may produce cytotoxins perforin, granzyme B, and TIA-1. However, NK/T-cell lymphoma with CD56 expression represents the most common immunophenotype among these tumors [8]. However, several studies have demonstrated that $\mathrm{CD} 3 \varepsilon+, \mathrm{CD} 56-$, EBV+, and cytotoxic molecule + cases can be also nasal type NK/T-cell lymphomas because these cases show a similar clinical course as in cases with CD56 expression. This marker, although it is highly useful for these lymphomas, is not specific and can be expressed in other peripheral T-cell lymphomas that express the $\gamma \delta$ T-cell receptor. Moreover, this diagnosis should not be made in the absence of cytotoxic molecule expression or EBV positivity. In this report, a case with CD56 expression
(Case 1) and other CD56- (Case 2) could be seen; both cases are $\mathrm{EBV}+$ and granzyme $\mathrm{B}+$, thus the phenotype was concordant with these criteria.

NK/T-cell lymphomas may disseminate quickly to several organs and the symptomatology will depend on the site of involvement. In patients with rapid dissemination and affectation outside the nasal cavity, this tumor is highly aggressive, with short survival times and poor response to therapy, as occurred in our cases. In these situations, in order to carry out a final and precise diagnosis and to improve the knowledge of true incidence and clinical symptomatology, an autopsy is necessary. The lymphomatous infiltrate in several organs might show an angiocentric and angiodestructive growth pattern. Also, extensive necrosis and vascular destruction are very common. In these cases, the differential diagnosis should be established with necrotizing granulomatous lesions such as tuberculosis and aspergillosis, with pancreatitis/penniculitis, vasculitis and histiocytosis, and finally with other kinds of lymphomas such as angiocentric, high-degree extranodal lymphomas, as well as NK/T 
panniculitis-like lymphomas. In Case 1, we also carried out the differential diagnosis with nontumoral lesions such as necrotizing pancreatitis and with tumoral lesions like pancreatic adenocarcinomas due to the high level of $\mathrm{Ca}$ 19.9 and the ductus pancreatic distortion by necrotic and inflammatory lesions. Curiously, in our two cases, the initial presumptive diagnosis was tuberculosis.

To conclude, NK/T-cell lymphomas are tumors mainly caused in Asian and South American countries, but can also be produced in the Western population. These tumors mainly involve the nasal cavity although in some cases this area cannot be involved, as in our reports, so it is necessary to keep in mind this fact in order to suspect the diagnosis. Several cases can disseminate outside the nasal cavity and multiple organs could be infiltrated; these cases are more aggressive than those localized in the nose and the response to treatment is poor. The mortality is high in these lymphomas and an autopsy could be necessary to carry out the correct diagnosis. This usually requires a multimodality approach correlating clinical, morphological, inmmunophenotypic, and molecular data.

\section{Acknowledgment}

The authors would like to acknowledge Ms. Angela Barnie for the English correction of this manuscript.

\section{References}

[1] J. K. C. Chan, E. S. Jaffe, and E. Ralfkiaer, "Extranodal NK/Tcell lymphoma, nasal type," in Pathology and Genetics: Tumours of Haematopoietic and Lymphoid Tissues, E. S. Jaffe, N. L. Harris, H. Stein, and J. W. Vardiman, Eds., World Health Organization Classification of Tumours, pp. 204-207, IARC Press, Lyon, France, 2001.

[2] F. Aydin, P. M. Bartholomew, and D. G. Vinson, "Primary Tcell lymphoma of the brain in a patient at advanced stage of acquired immunodeficiency syndrome," Archives of Pathology and Laboratory Medicine, vol. 122, no. 4, pp. 361-365, 1998.

[3] F. Cobo, P. Talavera, H. Busquier, and Á. Concha, "CNK/T-cell brain lymphoma associated with Epstein-Barr virus in a patient with AIDS," Neuropathology, vol. 27, no. 4, pp. 396-402, 2007.

[4] L. S. Young and A. B. Rickinson, "Epstein-Barr virus: 40 years on," Nature Reviews Cancer, vol. 4, no. 10, pp. 757-768, 2004.

[5] P. Porcu, R. A. Baiocchi, and C. Magro, "Recent developments in the biology and therapy of T-cell and natural killer-cell lymphomas," Current Opinion in Oncology, vol. 15, no. 5, pp. 353-362, 2003.

[6] J. P. Greer, M. C. Kinney, and T. P. Loughran Jr., "T cell and NK cell lymphoproliferative disorders," Hematology, vol. 2001, no. 1, pp. 259-281, 2001.

[7] Y. L. Kwong, A. C. L. Chan, R. Liang, et al., "CD56 NK lymphomas: clinicopathological features and prognosis," British Journal of Haematology, vol. 97, no. 4, pp. 821-829, 1997.

[8] M. M. C. Cheung, J. K. C. Chan, W. H. Lau, et al., "Primary non-Hodgkin's lymphoma of the nose and nasopharynx: clinical features, tumor immunophenotype, and treatment outcome in 113 patients," Journal of Clinical Oncology, vol. 16, no. 1, pp. 70-77, 1998.
[9] S. B. Ng, K. W. Lai, S. Murugaya, et al., "Nasal-type extranodal natural killer/T-cell lympholmas: a cliniclopathologic and genotypic study of 42 cases in Singapore," Modern Pathology, vol. 17, no. 9, pp. 1097-1107, 2004. 